\title{
COSMETICS AND THEIR ASSOCIATED ADVERSE EFFECTS: A REVIEW
}

\author{
Khan AD ${ }^{* 1}$, Alam MN ${ }^{1}$ \\ Assistant Professor, Ram-Eesh Institute of Vocational and Technical Education, Greater Noida, Uttar Pradesh, India.
} \begin{abstract}
The word 'cosmetics' is taken from a Greek word "kosmeticos" which means to adorn. Since early days materials used for beautification or improvement of appearance comes under the category of cosmetics. People want to look beautiful and the concept of cosmetics is as old as mankind and civilization. The urge to beautify one's own body and look beautiful has been an urge in the human race since the tribal days. Assorted beauty products such as skincare products, hair products, fragrances, oral hygiene, and nail products, which may contain toxic chemicals that can be harmful to health are used especially by women. Since long time cosmetics have been known to enhance the appearance of the human body. In a society obsessed with beauty, people are lured to fake their appearance as a cure for their insecurities. The estimated value of cosmetic industry today is around 20 billion dollar globally. As a consumer, we are constantly attracted in using beauty and personal care products. But these products, which are supposed to make us feel healthy and look beautiful, have a deep dark side. Various toxic ingredients and hazardous chemicals used in cosmetics are incorporated in beyond acceptable limits. These chemicals may cause serious ill effects on skin and may also enter skin and other organs causing carcinogenicity. Cosmetics have not only seeped into the fashion world but are also playing a prominent role in one's day-to-day life. Thus, it becomes a necessity to make people aware of various harmful effects of cosmetics and chemicals used in cosmetics.
\end{abstract}

Keywords: Health, Skin, Hazards, Cosmetics, Chemicals, Heavy Metals.

Article info:

Received: December 03, 2018

Revised: January 16, 2019

Published Online: April 15, 2019

DOI: https://doi.org/10.31069/japsr.v2i1.1
Correspondence:

Azhar Danish Khan

Assistant Professor, Department of Pharmacy, Ram-Eesh Institute of Vocational \& Technical Education, Greater Noida, Uttar Pradesh, India. Phone: +91-8802235141

Email: $\underline{\text { azhardk@gmail.com }}$

\section{INTRODUCTION}

Cosmetics are products applied to the body for the purpose of beautifying, cleansing or improving appearance and enhancing attractive features. ${ }^{[1]}$ Cosmetics consist of a range of products such as tooth paste, shampoo, conditioners, mascara, after shave lotion, styling gel, creams, lotions, powders, perfumes, lipsticks, fingernail and toenail polish, eye and facial make-ups, hair wavers, hair dyes, hair sprays, deodorants and antiperspirants. The word 'make up' is defined as a cosmetic which refers primarily to colored products intended to alter the user's appearance. ${ }^{[2]}$ Schneider et al defined skincare products or cosmetics as mixtures of synthetic or natural chemical compounds used to improve the appearance or smell of the body. ${ }^{[3]}$ They are articles intended to be rubbed, poured, sprinkled or sprayed on, introduced into, or otherwise applied to the human body or any part thereof for promoting attractiveness, cleansing, beautifying or altering the appearance without affecting the body structure or functions

\section{Historical significance of Cosmetics}

The Science of cosmetology is believed to have originated in ancient world in countries like Egypt and India, but the earliest records of cosmetic substances and their application dates back to
Circa 2500 and 1550 B.C, to the Indus valley civilization. ${ }^{[4]}$ An old remedy for cracked lips is found in history. Cracked lips, besides being painful, spoil the beauty of the face. The rind of Bel fruit (Aegle marmelos Corr.) is powdered and mixed in woman's milk and the paste thus prepared is applied to the cracked lips. The cracking will stop and the cracks will heal within 10 days the Superfluous hair was considered to be a mark of disgrace and a large number of depilatory agents were recommended to get rid of it. Dried fruits of Aavalakatti (Emblica officinalis Gaertn.) and dried fruits of Pimpali (Piper longum Linn.) were used. The mixture was soaked in the milky latex of Nivadunga (Cactus: Euphorbia nivulia Ham.) This compound was applied to the desired place, the hairs from that area were found to fall off". ${ }^{[5]}$

The use of kajal has a long history in Hindu Culture. Various beauty rituals in the present day rituals such as coloring of hair, depilation and exfoliation find their roots in ancient Egyptian culture. ${ }^{[5]}$ In earlier days, the Romans, Greeks and Egyptians used various cosmetics which contain white Lead and Mercury. ${ }^{[6]}$ Oils with essence and ointments were used to clean and soften the skin and mask body odor while dyes and natural paints were used to color the face, mainly for ceremonial and religious occasions. ${ }^{[7]}$ 


\section{Khan et al, Cosmetics and Adverse Effects}

There was a common ancient belief that eye makeup could remove evil spirits and improve sight. ${ }^{[6]}$

\section{Common Cosmetics Products and associated toxicities}

Some factors such as advertisement, peer pressure and social acceptance, influence the choice of skincare products applied by most women. Robertson et al conducted a study and reached a conclusion that women who use make-up have some sense of insecurity, and are anxious and lack confidence about them. ${ }^{[8]}$ Cosmetic products contain various harmful or toxic chemicals capable of causing harmful effects to the skin. Manufacturers of Cosmetic products do not only use synthetic ingredients but also natural products, such as Shea butter, Rose extract, and cane sugar which are sustainable, cheap and less harmful to the consumer. ${ }^{[9}$ Skincare products such as perfumes, make up, nail polish etc, remain on the skin for a longer period of time and can cause harmful effects like allergic reactions. ${ }^{[10]}$ Moisturizers increase the hygroscopic properties of the skin particularly when used in high concentration. It can cause irritation and exfoliation

\section{Skin Lightening agents}

Skin lightening agents such as hydroquinone (HQ) is found to be one of the most harmful chemicals. There have been found reports of ochronosis and potential mutagenicity. Ochronosis is an uncommon adverse effect of $\mathrm{HQ}$, with features like progressive darkening of the area to which the cream containing high concentrations of HQ is applied for many years. Hydroquinone is a hydroxyphenolic compound that inhibits the synthesis of melanin by inhibiting tyrosinase enzyme $\mathrm{t}$ may also function by interfering with the formation or degradation of melanosomes and by inhibiting the synthesis of DNA and RNA within melanocytes. Hydroquinone is a most commonly used depigmenting agent at present, but it is found to be highly cytotoxic to melanocytes and potentially mutagenic to mammalian cells. ${ }^{[1]}$ It causes irritation redness and burning, also causing exogenous ochronosis. Ochronosis may result in loss of elasticity of the skin and impaired wound healing which has resulted in a ban on its over-the-counter use in America and many other countries. It was allowed only for small areas of skin and for treating things like age spots or sun spots. ${ }^{[12]}$

\section{Black Henna}

Black henna is the combination of red henna with pphenylenediamine (PPD), and is used for temporary 'black henna tattoos .Black henna tattoo is a chemical stain due to the presence of p-phenylenediamine (PPD), available in the form of commercial hair dye mixed into the henna paste. PPD is added to henna to accelerate the dyeing and drying process (to only $30 \mathrm{~min}$ ), to strengthen and darken the color, to enhance the design pattern of the tattoo, and to make the tattoo last longer Negative effects of PPD include blisters, surface oozing, swelling and erythromatous rashes on the skin. Studies have been done and reports given about the immediate allergic reactions on using henna dyes. Various cases involving sneezing, runny nose, cough and shortness of breath instead of skin reaction have been found. ${ }^{[13][14]}$ There have been some reports of the appearance of localized hypertrichosis after black henna tattoos without allergic reactions to the tattoo. ${ }^{[15]}$ Many cases of toxicity from the black powder were noted in Sudan in early $80 \mathrm{~s}$ which were used for body painting, some cases were found to be fatal,. The initial symptoms are massive edema of the face, lips, glottis, pharynx, neck, and bronchi, occurring within hours of application of the dye mix to the skin, and sometimes requiring emergency tracheotomy for respiratory obstruction. The symptoms were found to progress on the second day to anuria and acute renal failure. Dialysis helped some patients, but others have died from renal tubular necrosis. ${ }^{[16]}$

\section{Sunscreen products}

Sun-screening agents used nowadays can cause irritant, allergic, phototoxic or photo-allergic reactions. The most common sensitizers are Benzophenones. While debenzoyl methanes, paraaminobenzoic acid (PABA) and cinnamates may cause photoallergic dermatitis. ${ }^{[17]}$ The allergic reactions which are related with deodorant/anti perspirants and fragrances are mostly caused by the fragrance or other ingredients. Fragrances may enter the body through skin(adsorption), lungs, air ways, ingestion and through pathways from the nose directly to the brain and can cause headaches, dizziness, fatigue, irritation to eyes, nose and throat, forgetfulness and other symptoms. When used the fragrances are sprayed in the air or found in air can cause air-borne contact dermatitis. Coumarins, phethleugenol which are usually found in fragrances are suspected carcinogens, while phthalates are suspected hormones disrupters. ${ }^{[18]}$

\section{Shampoos}

The contact time of shampoos and conditioners with the skin is very less; they are only applied to the hair and as such, thus causing less adverse effects. However, the problem arises when they come in contact with the eyes during washing of the hair. The most common effect of using shampoo is the matting of the scalp hair also referred to as tangling of hair. ${ }^{[19]}$ Another factor to be considered is $\mathrm{pH}$ of the shampoo. Most of the shampoos have an alkaline $\mathrm{pH}$, which causes hair shaft swelling, making the hair liable to damage. A shampoo with neutral $\mathrm{pH}$ is the best choice for chemically treated hair from either permanent dyeing or permanent waving. ${ }^{[20]}$ When the critical assessment of validated data on the frequency of contact allergies to shampoo was done, it showed their low risk. ${ }^{[21]}$ Given that shampoos are diluted by water, have a short contact time, and are rinsed off, the risk of sensitization is highly unlikely. ${ }^{[22]}$ Active ingredients in hair bleaching product such as Hydrogen peroxide solutions, and Ammonium persulfate, may cause Types I and IV allergic contact reactions.

Health hazards associated with chemicals used in formulation of Cosmetics

\section{$B H A$ and $B H T$}

BHA (butylated hydroxyl anisole) and BHT (butylated hydroxyl toluene) are closely related synthetic chemicals used as preservatives in moisturizers and lipsticks, among other cosmetics.BHA and BHT can cause allergic reactions in the skin. BHA has been classified as a possible human carcinogen by the 


\section{Khan et al, Cosmetics and Adverse Effects}

International Agency for Research on Cancer. The European Commission on Endocrine Disruption has also mentioned BHA as a Category I priority substance, based on evidence that it interferes with hormone function. ${ }^{[23]}$ BHT may act as a tumor promoter in certain situations. Little evidence suggests that high doses of BHT may mimic estrogen, the primary female sex hormone, and prevent expression of male sex hormones, resulting in adverse reproductive affects.

\section{Coal Tar Dyes}

Coal tar is a made up of many chemicals obtained from petroleum. Colors derived from coal tar are used mostly in cosmetics, generally identified by a five digit Color Index (CI) number. The p-phenylenediamine is a common coal tar dye used in many hair dyes. Phenylenediamine is found more in darker hair dyes than lighter colours. Adverse reactions caused by p-phenylenediamine are stinging sensations, with an erythromatous rash, swelling, blisters, and surface oozing. Various reports have been found in the literature of immediate allergic (and also anaphylactic) reactions on using henna dyes. ${ }^{[24]}$ Most cases have sneezing, runny nose, cough, and shortness of breath instead of skin reactions. Coal tar may also be associated with cancer and the main concern with individual coal tar colors (whether produced from coal tar or synthetically) is the possibility of them to cause cancer. These colors may be found contaminated with low levels of heavy metals and some are combined with Aluminum substrate. Aluminum compounds and many heavy metals may cause adverse effects to the brain. Some of these colors used to produce these dyes are not approved as food additives, yet they are used in cosmetics that may be ingested, like lipstick. P-phenylenediamine has been found to be carcinogenic. ${ }^{[25]}$ It has been established that women who use hair dyes especially over a long period have increased risk of developing non-Hodgkin's lymphoma (cancer of the lymph system). The European Union has classified p-phenylenediamine as toxic (whether by contact, inhalation, or by ingestion) and as very toxic to aquatic organisms, as it may cause long-term adverse (chronic) effects in the aquatic environment. ${ }^{[26]}$

\section{DEA (Cocamide DEA and Lauramide DEA)}

DEA (diethanolamine) related ingredients are used to make cosmetics creamy or sudsy, or as a $\mathrm{pH}$ adjuster to reduce the acidity of other ingredients. They are found in shampoos, soaps and cleansers.DEA reacts with nitrites in cosmetics to form nitrosamines. Nitrites are occasionally added to products as anticorrosive agents or can be present as contaminants. ${ }^{[27]}$ The degradation of some chemicals used as preservatives in cosmetics can release nitrites when the product is exposed to air. During experiments conducted in laboratory, exposure to high doses of DEA-related ingredients has been shown to cause liver cancers and precancerous changes in skin and thyroid. These chemicals may also cause mild to moderate skin and eye irritation. Cocamide DEA is found to be hazardous to the environment because of its acute toxicity to aquatic organisms and can cause bioaccumulation. ${ }^{[27]}$ Cocamide-DEA was listed under carcinogenic toxicant in June 2012. The substance is generally used as a foaming agent in soaps, shampoos, hair dye, cosmetics and household cleaning formulas. Cocamide DEA works as a surfactant, which means it helps soaps and shampoos to lather and foam. If a surfactant is too strong it can strip away your body's natural oils, leaving your skin dried out. If this natural protection against microbes and other environmental factors is not there your skin might get flaky and itchy. Your skin also becomes more prone to infection. Other examples of strong surfactants that can cause dry skin include sulfates, commonly sodium laureth sulfate and sodium lauryl sulfate, and betaines (usually cocamidopropyl betaine). Apart from drying your skin out (which we all know is bad enough), Cocamide DEA has been implicated in much more serious and long-term health risks. Cocamide DEA can complex with preservatives to form very dangerous chemicals called nitrosamines. One such example is called NDELA, a compound that has been shown to cause cancer in rats. NDELA is readily absorbed through the skin, especially when it's applied to large areas of your body. They're usually found in less expensive skin care products, but nothing is more valuable than your health.

\section{Di butyl Phthalate (DBP)}

DBP is mainly used in cosmetics for nails as a solvent for dyes and as a plasticizer that prevents nail polishes from becoming hard and brittle. It has been shown to cause developmental defects, changes in the prostate and testes and reduces sperm counts. ${ }^{[28]}$ It has also been found that it act as a suspected endocrine disruptor on the fact that it interferes with hormone function, and may cause harm to the unborn child and worsen infertility. Various researches reveal that exposure to phthalates may cause serious health effects such as liver and kidney failure in young children when products containing phthalates are ingested for extended periods. ${ }^{[29][30]}$ It has been found that phthalates reduce sperm count in men and reproductive defects in the developing male foetus (when the mother is exposed during pregnancy), among other health effects.

\section{Parabens}

For protecting cosmetics from microbial contamination, preservatives are used. The most commonly used preservative in cosmetics are parabens. Around 75 to 90 per cent of cosmetics contain parabens (typically at very low levels). Parabens easily permeate the skin and are suspected of interfering with hormone function (endocrine disruption). They mimic oestrogens, the primary female sex hormone. They may also interfere with male reproductive functions. Various studies indicate that methylparaben applied on the skin reacts with other chemicals, leading to increased skin aging and DNA damage. ${ }^{[31]}$ Certain foods, such as barley, strawberries, carrots, onions currents, and vanilla, also contain Parabens. ${ }^{[32]}$ Parabens in foods are metabolized when eaten, making them less strongly estrogenic. On the other side when applied to the skin and absorbed into the body, parabens in cosmetics bypass the metabolic process and enter the blood stream and body organs intact. It has been determined that women are exposed to $50 \mathrm{mg}$ per day of parabens from cosmetics. ${ }^{[32]}$ They are associated with cancer and neurotoxicity among other adverse health effects. ${ }^{[33]}$

Perfume (Fragrance)

Perfume is a mixture of essential oils or aromatic compounds and solvents, used to give the human body, animals, 


\section{Khan et al, Cosmetics and Adverse Effects}

food, objects, and living-spaces an pleasant scent. It is found usually in liquid form and used to give a pleasant scent to an individual's body. Perfumes are also used in cosmetics. Around 3,000 chemicals are used as fragrances. Fragrance is a main ingredient in perfumes, deodorants and colognes. Almost all the cosmetic products contain perfumes. Even if these products are marketed as "fragrance-free" or "unscented" may contain fragrance ingredients in the form of masking agents that prevent the brain from perceiving their odor. ${ }^{[34]}$ Many of fragrance ingredients which are unlisted are irritants and can cause allergies, severe headache and asthma symptoms. Perfume can worsen asthma and perhaps even contribute to its development in children. It is ranked the second most common cause of allergy in patients. [35]

Polyethylene Glycols (PEGs)

Polyethylene glycols (PEGs) are compounds based on petroleum that are widely used in creams as thickeners, solvents, softeners and moisture-carriers. During the production processes, PEGs may get contaminated with detectable amounts of 1, 4-dioxane. This 1, 4- dioxane is found to be causing cancer. It does not easily degrade and can remain in the environment long after it is rinsed down the shower drain. ${ }^{[18]}$ PEGs also show some evidence of genotoxicity and can cause irritation and systemic toxicity if used on broken skin.

\section{Petrolatum}

Petrolatum acts as a barrier to entrap moisture in the skin in a variety of moisturizers. It is used in hair care products to make them shine. It is also known as mineral oil jelly. Polycyclic aromatic hydrocarbons (PAHs) may be found as a contaminant in Petrolatum. It is found in various studies that after a long term exposure to PAHs it may be associated with cancer. On this basis, the European Union categorize petrolatum as a carcinogen and restricts its use in cosmetics. PAHs in petrolatum may also cause allergies and skin irritation. ${ }^{[36]}$

\section{Siloxane}

Siloxanes are silicone-based compounds used in various cosmetic products to make them soft and smooth. They make hair products dry faster and enhance the spreadability of deodorant creams. They are most often used in moisturizers and facial treatments. The commonly used Siloxane which are toxic are cyclotetrasiloxane and cyclopentasiloxane. They have the potential of bioaccumulating in aquatic organisms. Cyclotetrasiloxane is a type of endocrine disruptor, as it interferes with human hormone function, and as possible reproductive toxicant, may also impair human fertility. ${ }^{[37]}$

\section{Health risks Associated with Heavy Metals in Cosmetics}

Heavy metals have been involved in cosmetics commonly used among women. ${ }^{[38]}$ Harmful effects of heavy metals in various cosmetics products like facial make-up have been reviewed in

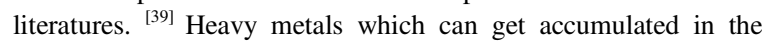

body over a period of time are known to cause various health problems. Some of the health risks associated with heavy metals in cosmetics are cancer, reproductive and developmental disorders, neurological problems, cardiovascular, skeletal, blood, immune system, kidney and renal problems, headaches, vomiting, nausea and diarrhea, lung damage. They may also cause contact dermatitis; and brittle hair and hair loss. Some heavy metals are hormone disruptors while others are respiratory toxins. They can enter the body through ingestion or absorbed through the broken skin. ${ }^{[40]}$

Cadmium

Cadmium is found naturally in the environment. Cadmium present in body and hair creams are absorbed into the body through dermal contact stored in the kidney and the liver, although it can be found in almost all adult tissues. According to IARC, it is considered to be "carcinogenic to humans and its compounds, categorized as known human carcinogens by the United States Department of Health and Human Services. ${ }^{[41]}$ If ingested in high levels, it may lead to severe stomach irritation, vomiting and diarrhea, while exposure to lower levels for a long time can lead to kidney damage, deformity of bones, and weakens the bones so that they break easily.

Lead

Lead can be found as impurity in Lipsticks via the use of contaminated raw materials or through the use of pigments that may contain lead. ${ }^{[42] . ~ S k i n ~ c o n t a c t ~ w i t h ~ L e a d ~ o c c u r s ~ d a i l y, ~ a n d ~}$ some amount has been found to be absorbed through the skin. The use of leaded eye powders (e.g., Surma, Kohl) has been associated with increased blood-lead levels in children and women. ${ }^{[43]}$ Pregnant women and young children are at higher risk because it can cross the placenta with ease and enter the brain of the fetus. It can also be transferred to infants through milk of lactating mothers and stored in bones. ${ }^{[44]}[45]$ Lead exposure has been found to cause miscarriage, hormonal changes, reduced fertility in men and women, menstrual irregularities, delays in puberty onset in girls. ${ }^{[42]}$ Lead and inorganic Lead compounds have been classified as a suspected carcinogenic to humans. ${ }^{[40]}$

\section{Nickel}

Nickel is found in abundance in nature, everyone is exposed to small amounts, mostly through food, air, portable water, soil, household dust, and skin contact with products containing it, including cosmetics. ${ }^{\left[{ }^{[3]}\right.}$ High levels of exposure can lead to serious health effects depending on route and the kind of nickel exposed to. ${ }^{[43]}$ While certain types of Nickel are considered to be "toxic" because of their carcinogenic effect, metallic Nickel and alloys have been classified as possibly carcinogenic to humans. ${ }^{[40]}$ Nickel can also be found allergic is also and it can cause severe contact dermatitis. ${ }^{[43]}$ The first case of Nickel allergy caused by eye shadow has been reported; even as $1 \mathrm{ppm}$ of it may trigger a preexisting allergy. ${ }^{[46]}$

Mercury 


\section{Khan et al, Cosmetics and Adverse Effects}

Mercury is a common ingredient present in skin-lightening soaps and creams. It is also found in various other cosmetics, such as eye make-up, cleansing products and mascara. Skin-lightening soaps and creams are widely used in certain African and Asian countries. ${ }^{[47]}$ Mercury salts works by inhibiting the formation of melanin, thus making the skin lighter in tone. Mercury is found in cosmetics two forms viz. inorganic and organic. Inorganic mercury (e.g. ammoniated mercury) is used as skin-lightening agents in soaps and creams. Organic mercury compounds (ethyl mercury and phenyl mercuric salts) are used as preservatives in cosmetics like eye make-ups, cleansing products and mascara. The main adverse effect associated with inorganic mercury contained in skin lightening soaps and creams is kidney damage. ${ }^{[48]}$

Mercury when present in skin-lightening products may also cause skin rashes, skin discoloration and scarring, as well as a reduction in the skin's resistance to bacterial and fungal infections. Other side effects include anxiety, depression or psychosis and peripheral neuropathy. Mercury from soaps, creams and other cosmetic products is eventually discharged into wastewater. The mercury then enters the environment, where it becomes methylated and enters the food-chain as the highly toxic methyl mercury in fish. Pregnant women when eat fish containing methyl mercury, it is transferred to their foetus, which can lead to neuro-developmental deficits in the children. ${ }^{[49]}$

\section{CONCLUSION}

The cosmetic products may present health risks and recurrent adverse effects are attributed to the toxic substances commonly found in their formulations. Although the various structures for the regulation and quality control of cosmetics around the world are quite complex and comprehensive, they should be more rigorous in the inclusion of new substances with toxic potential in the formulation of cosmetics to avoid damages to human health. To encourage improvements in the manufacture, marketing and use of cosmetic products by the population, it is necessary to apply a unified cosmeto-vigilance around the world. This public health strategy are a genuine means of obtaining information on the safety of cosmetic products and their ingredients, preventing the risks associated with the use of cosmetics become a serious public health problem.

\section{ACKNOWLEDGEMENT}

Authors are extremely thankful to the referred authors for their incredible findings that helped us in compiling this exhausted review article.

\section{CONFLICT OF INTEREST}

The author declares that they have no competing interests.

\section{REFERENCE}

1. Malik Vijay. The Drug and Cosmetics Act, 1940, $18^{\text {th }}$ Edition, New Delhi: Eastern Book Company. pp 5-6.
2. Draelos ZD. Cosmetics: The Medicine of Beauty. Journal of Cosmetic Dermatology. 2015;14 (2):91

3. Schneider G, Gohla S, SchreiberJ, Kaden W, Schomock U, Lewerkuhne HS, Kuschel A, Petsitis X, Pape W, Ippen H, Diembeck W. In-Skin cosmetics, Ullmann's Encyclopedia of Industrial Chemistry. $6^{\text {th }}$ Vol., Germany: Wiley VCH, 2001, 10, pp. 24-29.

4. Lal BB. The Saraswati Flows on: The Continuity of Indian Culture. 2002, Vol II. p. 56-57

5. Rao SA. Krishnaswami Mahadick ed.- Sri Sarabhendra Vaidya Ratnavali. (Saraswati Mahal Library, Tanjore; 1952. pp. 277-381.

6. A. Claeyssens. The History of Cosmetics and Make up, 2009. [Online]. Available: http://ezinearticles.com/?The -History-of-Cosmetics-\&-makeup\&id=1857725.

Accessed: February 28, 2018.

7. M. Price. Cosmetics, Styles \& Beauty Concepts in Iran, 2001. [Online]. Available: http://www.iranchamber. com/culture/articles/cosmetics_beauty.php. Accessed: February 20, 2018.

8. Robertson J, Fieldman G, Hussey TB. "Who wears cosmetics?" Individual differences and their relationship with cosmetic usage. Individual Differences Research. 2008; 6(1): 38-56.

9. Rinaldi A, Healing beauty? More biotechnology cosmetic products that claim drug-like properties reach the market," EMBO Reports. 2008; 9(11): 1073-1077.

10. Dooms-Goosens A. Cosmetics as causes of allergic contact dermatitis .Cutis. 1993; 52(5):316-320

11. Donsing P, Viyoch J. Thai Breadfruit's Heartwood Extract: A New Approach to Skin Whitening. Srinakharinwirot Science Journal. 2008; 24 (1): 9-23.

12. Gabriel J. Hydroquinone: Cancer-Causing Skin Bleach, 2008. [Online]. Available: http://thegreenbeautyguide. com. Accessed: February 20, 2018.

13. Nigam PK, Saxena AK. Allergic contact dermatitis from henna. Contact Dermatitis, 1988; 18(1):55-56.

14. Spornraft-Ragaller P, Kammerer E, Gillitzer C, Schmitt J. Severe allergic reactions to para-phenylenediamine in children and adolescents: should the patch test concentration of PPD be changed? Journal Deutsche Dermatologische Gesellschaft. 2012;(10): 258-263

15. Durmazlar SP, Tatlican S, Eskioglu F. Localized hypertrichosis due to temporary henna tattoos: report of three cases. Journal of Dermatological Treatment. 2009; 20(6): 371-373

16. D'Arcy PF. Fatalities with the use of henna dye. Pharmacy International. 1982;(3): 217-218

17. Johansen JD, Rastogi SC, Menne T, Threshold responses in cinnamic-aldehyde-sensitive subjects: Results and methodological aspects, Contact Dermatitis. 1996; 34(3):165-171.

18. Bridges B. Fragrance: emerging health and environmental concerns. Flavour Fragrances Journal. 2002; 17(5):361-371.

19. Wilson CL, Ferguson DJ, Dawber RP. Matting of scalp hair during shampooing: A new look, Clinical and Experimental Dermatology, 1990; 15(2):139- 142.

20. Draelos ZD. Shampoos, conditioners, and camouflage techniques. Dermatologic Clinics. 2013; 31(1):173178. 


\section{Khan et al, Cosmetics and Adverse Effects}

21. Adams RM, Maibach HI. A five-year study of cosmetic reactions. Journal of the American Academy of Dermatology. 1985; 13(6):1062-1069.

22. Fewings J, Menné T. An update of the risk assessment for methylchloroisothiazolinone/methylisothiazolinone (MCI/MI) with focus on rinse-off products. Contact Dermatitis. 1999; 41(1): 1-13.

23. Schrader TJ, Cooke GM. Examination of selected food additives and organochlorine food contaminants for androgenic activity in vitro. Toxicological Sciences. 2008; 53(2): 278-288.

24. Nigam PK, Saxena AK. Allergic contact dermatitis from henna. Contact Dermatitis, 1988; 18(1):55-56.

25. Rollison DE, Helzlsouer KJ,Pinney SM.Personal hair dye use and cancer: a systematic literature review and evaluation of exposure assessment in studies published since 1992. Journal of Toxicology and Environmental Health Part B: Critical Review. 2006; 9(5):493-500.

26. Zhang Y. Personal use of hair dye and the risk of certain subtypes of non-Hodgkin lymphoma. American Journal of Epidemiology. 2008; 167(11):1321-1331.

27. Turkoglu M, Pekmezci E, Sakr A. Evaluation of irritation potential of surfactant mixtures. International Journal of Cosmetic Science. 1999; 21(6): 371-382.

28. Barlow NJ, McIntyre BS, Foster PM. Male reproductive tract lesions at 6,12 , and 18 months of age following in utero exposure to di(n-butyl) phthalate. Toxicologic Pathology. 2004; 32(1): 79-90.

29. Health Canada, Report on Human Biomonitoring of Environmental Chemicals in Canada: Results of the Canadian Health Measures, Survey Cycle 1 (20072009), Ottawa, 2010. [Online]. Available: https://www.canada.ca/en/health-canada/services/env ironmental-workplace-health/reports-publications/env ironmental-contaminants/report-human-biomonitoringenvironmental-chemicals-canada-health-canada-2010. html. Accessed: February 20, 2018.

30. Stahlhut RW, van Wijngaarden E, Dye TD, Cook S, Swan SH. Concentrations of urinary phthalate metabolites are associated with increased waste circumference and insulin resistance in adult U.S. males, Environmental Health Perspectives. 2007; 115(6):876-882.

31. Darbre PD, Aljarrah A, Miller WR, Coldham NG, Sauer MJ, Pope GS. Concentrations of Parabens in human breast tumours. Journal of Applied Toxicology. 2004; 24(1): 5-13.

32. Smith CN, Alexander BR. The relative cytotoxicity of personal care preservative systems in Balb/C 3T3 clone A31 embryonic mouse cells and the effect of selected preservative systems upon the toxicity of a standard rinse-off formulation. Toxicology in Vitro. 2005; 19(7):963-969.

33. Anderson RC, Anderson JH. Acute toxic effects of fragrance products, Archives of Environmental HealthAn International Journal. 1998; 53(2): 138-146.
34. Nardelli A, Drieghe J, Claes L, Boey L, Goossens A, , Fragrance allergens in 'specific' cosmetic products, Contact Dermatitis. 2011; 64(4): 212-219.

35. Park ME, Zippin JH. Allergic contact dermatitis to cosmetics. Dermatologic Clinics. 2014; 32(1):1-11.

36. Ulrich $\mathrm{G}$, Sensitization to petrolatum: an unusual cause of false-positive drug patch-tests. Allergy. 2004; 59(9): 1006-1009.

37. Curtis J, Colas A. Medical applications of silicones, in: B.D. Ratner, A.S. Hoff man, F.J. Schoen, and J. E. Lemons, eds., Biomaterials Science. New York: Elsevier/Academic Press, New York, USA, 2004, pp. 698-707,

38. Popoola OE, Bisi-johnson MA, Abiodun A, Ibeh OS, Heavy metal content and antimicrobial activities of some naturally occurring facial cosmetics in Nigeria. Ife Journal of Science. 2013; 15(3): 637-644.

39. Ramakant S, Poornima S, Sapina J, Mathur HB, Agarwal HC. Heavy metal in cosmetics, Centre for science and Environment. 2014; (45): 3-28.

40. Horii Y, Kannan K. Archives of Environmental Contamination and Toxicology. 2008; 55(4): 701-710.

41. Al-Dayel O, Hefne J, Al-Ajyan T. Human Exposure to Heavy Metals from Cosmetics. Oriental Journal of Chemistry. 2011; 27(1): 1-11.

42. Al-Saleh, I, Al-Enazi, S, Shinwari, N, Assessment of lead in cosmetic products. Regulatory Toxicology and Pharmacology, 2009; 54(2): 105-113.

43. Ababneth FA, Abu-Sbeih KA, Al-Momani IF. Evaluation of Allergenic Metals and Other Trace Elements in Personal Care Products. Jordan Journal of Chemistry. 2013; 8(3): 179-190.

44. Chauhan, SB, Chandak,A, Agrawal SS. Evaluation of Heavy Metals contamination in Marketed Lipsticks. International Journal of Advance Research. 2014; 2(4): 257-262.

45. Borowska S, Brzóska MM. Metals in cosmetics: Implications for human health. Journal of Applied Toxicology. 2015; 35(6): 551-572.

46. Zakaria,A, Ho YB. Heavy metals contamination in lipsticks and their associated health risks to lipstick consumers. Regulatory Toxicology and Pharmacology. 2015; 73(1): 191-195.

47. Biebl KA, Warshaw EM, Allergic contact dermatitis to cosmetics. Dermatologic Clinics. 2006; 24(2): 215-232.

48. Mehrdad RR, Mehravar RR, Sohrab K, Moghadamnia AA. Current approaches of the management of mercury poisoning: need of the hour. DARU Journal of Pharmaceutical Sciences. 2014; 22(1): 22-46.

49. Chan TY. Inorganic mercury poisoning associated with skin-lightening cosmetic products. Clinical Toxicology (Philadelphia). 2011; 49(10): 886-891. 